\title{
ON ARNOLD'S CONJECTURE FOR SYMPLECTIC FIXED POINTS
}

\author{
KAORU ONO \\ Department of Mathematics, Ochanomizu University, Otsuka, Tokyo 112, Japan \\ Current address: Hokkaido University, Sapporo, 060, Japan \\ E-mail: ono@math.sci.hokudai.ac.jp
}

1. Introduction. It is well-known that the number of critical points of a smooth function on a closed manifold is bounded below by the Lusternik-Schnirelmann category of the manifold. If all the critical points are non-degenerate, as a consequence of Morse theory, the number of critical points is at least as great as the sum of the Betti numbers and torsion numbers (namely, the minimal number of generators of the homology groups). Another origin of our story is the pioneering work of Poincaré, which is now called Poincaré's last geometric theorem or the Poincaré-Birkhoff fixed point theorem. Let $\phi$ be a self-diffeomorphism of an annulus $S^{1} \times[0,1]$ which is area and orientation preserving. If $\phi$ satisfies the twisting condition, i.e., $\phi$ maps $S^{1} \times\{0\}$ and $S^{1} \times\{1\}$ to themselves and "rotates" them in opposite directions, then the theorem states that there are at least two fixed points of $\phi$ (see for instance [2]). Arnold proposed to study the symplectic analog of critical point theory and suggested to explore new fields, now collectively called symplectic topology.

A typical example of a symplectic manifold is the cotangent bundle $T^{*} X$ of a smooth manifold $X$. For a function $f$ on $X$, its differential $d f$ gives a lagrangian submanifold in $T^{*} X$, namely the graph $\Gamma_{d f}$. The intersection of $\Gamma_{d f}$ and the zero section $O_{X}$ can be identified with the critical point set of $f$, so the estimate mentioned above gives a lower bound on the number of intersection points. One can therefore think of intersections of lagrangian submanifolds as a generalization of critical point sets of functions on a certain space.

Let $(M, \omega)$ be a symplectic manifold and $L$ a lagrangian submanifold. A diffeomorphism $\phi$ is called a symplectomorphism if $\phi$ preserves the symplectic structure, i.e., $\phi^{*} \omega=\omega$. If a diffeomorphism $\phi$ is the time-one map of a flow generated by a time dependent Hamiltonian vector field $X_{H_{t}}$ (see $\S 2$ ), it is called an exact symplectomorphism.

1991 Mathematics Subject Classification: Primary 58F05.

The paper is in final form and no version of it will be published elsewhere. 
By the Cartan formula for the Lie derivative, exact symplectomorphisms are certainly symplectomorphisms. In the case of the graph $\Gamma_{d f}$ in $T^{*} X$, it is the image of the zero section by the time-one map of a Hamiltonian flow. For a closed lagrangian submanifold $L$ in $(M, \omega)$, there exists a symplectomorphism from a tubular neighborhood of $L$ in $M$ to a tubular neighborhood of $O_{L}$ in $T^{*} L$ (Weinstein). Thus for a small exact deformation $\phi, \phi(L)$ can be identified with the graph of an exact 1-form on $L$. Here a "small exact deformation" is an exact symplectomorphism whose Hamiltonian function $H$ is $C^{2}$-small.

For an exact symplectomorphism $\phi$, one may expect the number of intersection points of $L$ and $\phi(L)$ is at least the minimal number of critical points of smooth functions on $L$ and it is at least the minimal number of critical points of Morse functions on $L$ provided that $L$ and $\phi(L)$ intersect transversally. This is not true in general and we need some additional conditions to show such an estimate. For instance, a small circle on a surface can be separated from itself by an exact symplectomorphism.

On the other hand, this estimate is established in some situations. Hofer [14] and Laudenbach and Sikorav [18] established a somewhat weaker estimate for the case of a zero section in the cotangent bundle of a closed manifold. A breakthrough was made by Floer when he introduced a "middle infinite dimensional (co)homology theory" for the symplectic action functional which is now called Floer homology [7]. Floer's construction has now been extended by Oh [25]. Chekanov established an estimate for exact symplectomorphisms with small Hofer norm instead of assuming some condition for the lagrangian submanifold [5].

Since the graph $\Gamma_{\phi}$ of a symplectomorphism $\phi$ is a lagrangian submanifold in $(M \times$ $M,-\omega \oplus \omega)$, we can consider an analogous problem for fixed points of $\phi$. Note that the fixed point set of $\phi$ is identified with the intersection of $\Gamma_{\phi}$ and the diagonal set $\Delta_{M}$ and that $\Gamma_{\phi}$ is the image of $\Delta_{M}$ by $i d \times \phi$. The following problem, called Arnold's conjecture for symplectic fixed points, is the theme of this survey. There are good survey articles [23], [29] up to 1990. We include here recent progress on the topic.

ConjeCture. Let $M$ be a closed symplectic manifold and $\phi$ an exact symplectomorphism. Then the number of fixed points is at least the minimal number of critical points of functions on $M$. Moreover, if all the fixed points are non-degenerate, i.e., 1 is not an eigenvalue for $d \phi$ at any fixed points of $\phi$, then the number is at least the minimal number of critical points of Morse functions on $M$.

Since this minorant is bounded from below by Lusternik-Schnirelmann category, and so also by the cup-length, in the general case and by the sum of Betti numbers and torsion numbers in the non-degenerate case, we also have a conjecture in terms of these topological invariants. For the estimate of fixed points of an exact symplectomorphism, it has been established in several cases. In the two dimensional case, this conjecture is proven. The result is due to Nikishin, Simon (2-sphere), Conley and Zehnder (torus) and Floer and Sikorav (oriented closed surfaces of higher genus). After these works, Floer gave an estimate for the case that $\pi_{2}(M)=0$ using Floer homology. In the non-degenerate case, there is some progress. Floer himself generalized the result to the monotone case [8] and Hofer and Salamon and the author exteneded the argument to the weakly monotone case [15], [26]. Recently, K. Fukaya and the author, G. Liu and G. Tian, Y. Ruan, H. 
Hofer and D. Salamon proved that the number is at least the sum of Betti numbers (rank of rational homology). A similar result for non-exact symplectomorphisms symplectic isotopic to the identity is also studied by Lê and the author [19].

For the degenerate case, there are results by Floer, Lê and the author, Schwarz for the cup-length estimate or quantum cup-length estimate [8], [20], [31]. However the conjecture has not yet been proven, even in its weak form, in general. During the present conference, ${ }^{1}$ Rudyak and Oprea announced an estimate for the original form of the conjecture in cases including $\pi_{2}(M)=0$.

2. Variational set-up. Let $(M, \omega)$ be a symplectic manifold and $h: M \rightarrow \mathbf{R}$ a smooth function. We define the Hamiltonian vector field $X_{h}$ of $h$ by $d h+i\left(X_{h}\right) \omega=0$. For a time-dependent Hamiltonian function $H: M \times \mathbf{R} \rightarrow \mathbf{R}$, we have a time-dependent Hamiltonian vector field $X_{H_{t}}$, where $H_{t}(x)=H(x, t)$. Denote by $\phi$ the time-one map of the flow generated by $X_{H_{t}}$. For such a $\phi$, the Hamiltonian function $H$ can be chosen as a one-periodic function, i.e., $H(x, t+1)=H(x, t)$. Then there is a one-to-one correspondence between fixed points of $\phi$ and one-periodic solutions of the equation

$$
\dot{x}(t)=X_{H_{t}} .
$$

This equation is the Euler-Lagrange equation for the following functional on the space of null-homotopic loops:

$$
\mathcal{A}_{H}(x)=-\int_{D^{2}} u^{*} \omega+\int_{0}^{1} H(x(t), t),
$$

where $u: D^{2} \rightarrow M$ is an extension of the loop $x: S^{1}=\partial D^{2} \rightarrow M$. The first term of the right hand side may depend on the homotopy class of $u$ bounding the loop $x$, hence this functional is only defined on a certain covering space of the space of null-homotopic loops. However, we shall first restrict ourselves to the simple situation $\pi_{2}(M)=0$ and leave the general case to later discussion. In such a case, $\mathcal{A}_{H}$ is defined on the space $\mathcal{L} M$ of null-homotopic loops.

If one can extend critical point theory, e.g., Morse theory, to $\mathcal{A}_{H}$, one may get some lower bound for the number of one-periodic solutions. We note a couple of difficulties arising in this project. The Hessian of $\mathcal{A}_{H}$ is a first order differential operator which has an infinite number of both positive and negative eigenvalues. Roughly, finite dimensional critical point theory studies the difference in topology of (sub)level sets of a function. However in our setting, "homotopy type" changes by attaching a handle of infinite index which is homotopically trivial when we cross a non-degenerate critical value, so one may not see this difference homotopically.

Another difference is that the equation for the gradient flow is ill-posed as an initial value problem and may not have solutions passing through given loops. Here is a rough comparison between Morse theory and Floer theory.

\footnotetext{
${ }^{1}$ The author is grateful to J. Oprea and A. Tralle, the organizers of the workshop "Homotopy and Geometry" held at the Banach Center in June 1997, for a stimulating and enjoyable atmosphere.
} 


\begin{tabular}{l|l}
\hline$f: M \rightarrow \mathbf{R}$ Morse function & $\mathcal{A}_{H}: \mathcal{L} M \rightarrow \mathbf{R}$ \\
\hline Crit $(f)$ the critical point set & 1-periodic solutions of $\dot{x}=X_{H_{t}}(x)$ \\
\hline Morse index & Conley-Zehnder index \\
\hline gradient flow lines & connecting orbits for $\mathcal{A}_{H}$ \\
connecting critical points & between one-periodic solutions \\
\hline Morse homology & Floer homology \\
\hline
\end{tabular}

This table is just the beginning of the theory and one may hope for more comparisons (e.g., [11]).

Conley-Zehnder [6] used finite dimensional reduction for $\mathcal{A}_{H}$ in the case of a torus $\mathbf{R}^{2 n} / \mathbf{Z}^{2 n}$ and proved the conjecture (see also [4]). The theory of pseudo-holomorphic curves was invented by Gromov who gave many applications in symplectic topology [13]. One of them is the existence of a fixed point of an exact symplectomorphism in the case that $\pi_{2}(M)=0$. Floer combined these ideas and constructed "middle infinite dimensional homology theory", which is now called Floer homology. We shall sketch the argument in the case that $\pi_{2}(M)=0$.

In order to define the gradient of a function, one needs a Riemannian metric. We need a little preparation to define a metric on $\mathcal{L} M$. Since the unitary group $U(n)$ is a maximal compact subgroup of the group of linear symplectic transformations on a $2 n$-dimensional symplectic vector space, the structure group of the tangent bundle of $(M, \omega)$ reduces to $U(n)$. Hence, $M$ admits an almost complex structure $J$.

An almost complex structure $J$ on $(M, \omega)$ is called compatible or $\omega$-calibrated, if the bilinear form given by $g_{J}(u, v)=\omega(u, J v)$ is a Riemannian metric. By reduction of the structure group, there exist compatible almost complex structures unique up to homotopy. Pick a compatible almost complex structure $J$, where $g_{J}$ is a Riemannian metric which induces an $L^{2}$-inner product on the tangent space of the loop space. In this way, we get a "metric" on $\mathcal{L} M$.

Computing the gradient of $\mathcal{A}_{H}$ with respect to this metric, we get

$$
\operatorname{grad} \mathcal{A}_{H}(x)=J \dot{x}+\nabla H_{t}(x),
$$

where $\nabla H_{t}$ is the gradient of $H_{t}$ with respct to $g_{J}$. We also compute formally its Hessian, which is a first order elliptic ordinary differential operator. A critical point is called nondegenerate, if its Hessian is non-degenerate.

In our case, non-degenerate critical points of $\mathcal{A}_{H}$ are exactly one-periodic solutions such that 1 is not a Floquet multiplier, i.e., 1 is not an eigenvalue of $d \phi$ at any fixed point, where $\phi$ is the time-one map of the corresponding Hamiltonian flow. In Morse theory, the index of a critical point is the number of negative eigenvalues of Hessian matrix. In our case, there are infinitely many negative eigenvalues (as well as positive eigenvalues). However, the relative index, i.e., the difference of indices at one-periodic solutions, can be defined by the spectral flow [1] of Hessian operators along a path joining two oneperiodic solutions. Moreover there is a so-called Conley-Zehnder index [30] so that the relative index is the difference of Conley-Zehnder indices. 
Formally, a minus gradient trajectory $\gamma$ of $\mathcal{A}_{H}$ is a path $\gamma: \mathbf{R} \rightarrow \mathcal{L} M$ satisfying

$$
\dot{\gamma}=-\operatorname{grad} \mathcal{A}_{H}(\gamma)
$$

Interpret the map $\gamma: \mathbf{R} \rightarrow \mathcal{L} M$ as $u: \mathbf{R} \times S^{1} \rightarrow M$. Then a gradient trajectory corresponds to a map $u$ satisfying

$$
\frac{\partial u}{\partial s}+J(u) \frac{\partial u}{\partial t}+\nabla H_{t}(u)=0 .
$$

So far, these computations are just formal. From now on, we take this as the definition of gradient trajectories of $\mathcal{A}_{H}$, which we call connecting orbits for $\mathcal{A}_{H}$. Denote by $\widetilde{\mathcal{M}}\left(x^{-}, x^{+}\right)$the space of connecting orbits joining $x^{-}$to $x^{+}$. The equation (1) is invariant under translations in the $s$ variable, hence $\mathbf{R}$ acts on $\widetilde{\mathcal{M}}\left(x^{-}, x^{+}\right)$. This action is free unless $x^{-}=x^{+}$.

As we noticed, we can compare the difference of indices of Hessian operator along a path $u$ joining two one-periodic solutions $x^{-}$and $x^{+}$. The Atiyah-Patodi-Singer index theorem implies that the spectral flow equals the index of the corresponding elliptic operator on the cylinder with a certain boundary condition. In our situation, the spectral flow equals the index of the linearization of (1) with asymptotic convergence to $x^{ \pm}$as $s$ tends to $\pm \infty$. Hence the dimension of the moduli space of solutions of (1) is given by the difference of Conley-Zehnder indices.

The gradient flow of $f$ is called of Morse-Smale type if stable manifolds and unstable manifolds intersect transversally. Suppose that a function $f$ is a Morse function and its gradient flow is of Morse-Smale type. The Morse complex of $f$ is generated by the critical point set as modules and its differential is defined by counting, with signs, gradient trajectories joining two critical points of index difference 1 . The resulting homology group is called Morse homology, which is isomorphic to the ordinary homology. Details can be found in [32].

The Floer complex is generated by one-periodic solutions. The Morse-Smale condition of the gradient flow is interpreted as the surjectivity of the linearization operator of (1). Let $C_{i}(H, J)$ be the free module generated by one periodic solutions with Conley-Zehnder index $\mu=i$. Denote by $\mathcal{P}(H)$ the set of one-periodic solutions which are null homotopic. The boundary homomorphism $\partial: C_{i}(H, J) \rightarrow C_{i-1}(H, J)$ is defined by:

$$
\partial x=\sum_{\mu(y)=i-1} m(x, y) y,
$$

where $m(x, y)$ is the cardinality of $\mathcal{M}(x, y)=\widetilde{\mathcal{M}}(x, y) / \mathbf{R}$ counted with sign. By the Gromov compactness argument, if a sequence in $\mathcal{M}(x, y)$ is uniformly bounded up to the first derivative, it converges locally uniformly (after reparametrization). In this case, the limit may be a tuple $\left(u_{1}, \ldots, u_{k}\right) \in \mathcal{M}\left(x, x_{1}\right) \times \cdots \times \mathcal{M}\left(x_{k-1}, y\right)$. Here $u_{1}, \ldots, u_{k}$ are not constant paths in the loop space. For a generic pair $(H, J)$, the linearization of (1) is surjective and $\widetilde{\mathcal{M}}(x, y)$ is a manifold of dimension $\mu(x)-\mu(y)$.

Suppose that $\mu(x)-\mu(y)=1$. Since $\mathcal{M}(x, y)$ has positive dimension unless $x=y$, one of $\mathcal{M}\left(x_{i}, x_{i+1}\right)$ has negative dimension, hence is empty, if $k \geq 2$. So the sequence is convergent. If the derivatives of the sequence are not uniformly bounded, the Gromov compactness argument implies that $J$-holomorphic bubbles appear from the sequence. In 
the case of $\pi_{2}(M)=0$, no $J$-holomorphic sphere exists. Hence $\mathcal{M}(x, y)$ consists of finitely many points. This manifold also carries a natural orientation (see [8], [9], [12]). When $\mu(x)-\mu(y)=1$, we compare this orientation with the action of $\mathbf{R}$. If these orientations are the same, we count the point by +1 , if not, -1 . In this way, the definition of the boundary homomorphism is established.

We can show that $\partial^{2}=0$ by studying $\mathcal{M}(x, z)$ with $\mu(x)-\mu(z)=2$. This manifold may not be compact and its ends correspond to $\mathcal{M}(x, y) \times \mathcal{M}(y, z)$ with $\mu(y)=\mu(x)-1=$ $\mu(z)+1$. The signed number of points in the boundary of a compact 1-dimensional manifold must be zero and this implies that $\partial^{2}=0$.

The resulting homology group is called Floer homology $H F_{*}(H, J)$ and we can show that the groups $H F_{*}\left(H_{i}, J_{i}\right)$ are isomorphic for generic pairs $\left(H_{i}, J_{i}\right), i=1,2$.

To prove this, we need to study the solution space of the following equation:

$$
\frac{\partial u}{\partial s}+J(u) \frac{\partial u}{\partial t}+\nabla H_{s, t}(u)=0
$$

where $H_{s}$ is a path in the space of one-periodic Hamiltonians such that $H_{s}=H_{1}$ for $s<-R$ and $H_{s}=H_{2}$ for $s>R$ for some constant $R>0$. The equation (2) is an $s$-dependent analog of the equation (1) and not invariant under translations in the $s$ variable.

A similar argument as that in the proof of $\partial^{2}=0$ gives a chain homomorphism $H F_{*}\left(H_{1}, J_{1}\right) \rightarrow H F_{*}\left(H_{2}, J_{2}\right)$. In fact, it is an isomorphism (see, for instance, [23]).

For computation, we take a $C^{2}$-small Morse function $f$ and consider a Hamiltonian $H(x, t)=f(x)$. If the solutions of (1) are $t$-independent, hence correspond to gradient trajectories of $f$, then $H F_{*}(f, J) \cong H_{*+n}(M)$. Once the surjectivity of the linearization is established, the conclusion follows easily. For surjectivity, see [10], [26]. For another approach to computation, see [27].

So far, we have considered the case $\pi_{2}(M)=0$. In general, we need to work on a covering space of $\mathcal{L} M$. Namely, we introduce

$$
\widetilde{\mathcal{L}} M=\left\{(x, u) \mid x: S^{1} \rightarrow M, u: D^{2} \rightarrow M \text { such that }\left.u\right|_{\partial D^{2}}=x\right\} / \sim,
$$

where $(x, u) \sim(y, v)$ if and only if $x=y$ and evaluations of $\omega$ and $c_{1}(M)$ with the 2-spherical cycle $u \sharp(-v)$ are zero. Here $-v$ is the mapping from the disk with opposite orientation. Then $p: \widetilde{\mathcal{L}} M \rightarrow \mathcal{L} M$ is a covering space with covering transformation group $\Gamma=\pi_{2}(M) / \operatorname{Ker} I_{\omega} \cap \operatorname{Ker} I_{c_{1}(M)} . I_{\omega}$ and $I_{c_{1}(M)}$ are homomorphisms from $\pi_{2}(M)$ to $\mathbf{R}$ corresponding to the cohomology classes $[\omega]$ and $c_{1}(M)$.

The action functional $\mathcal{A}_{H}$ is well-defined on $\widetilde{\mathcal{L}} M$. Write

$$
\widetilde{\mathcal{P}}(H)=p^{-1}(\mathcal{P}(H)) \subset \widetilde{\mathcal{L}} M .
$$

The Floer chain complex is generated by $\widetilde{\mathcal{P}}(H)$, on which the Conley-Zehnder index is well-defined. The boundary homomorphism is defined, in the same way as before, by counting solutions of (1). We call a solution $u$ a connecting orbit from $\left(x^{-}, u^{-}\right)$to $\left(x^{+}, u^{+}\right)$ if the evaluations of $\omega$ and $c_{1}(M)$ on the 2 -spherical cycle $u^{-} \sharp u \sharp\left(-u^{+}\right)$are zero. Note that the number of end points $\left(x^{+}, u^{+}\right)$of connecting orbits, with index difference 1 , starting from $\left(x^{-}, u^{-}\right)$can be infinite. However the boundary homomorphism is well-defined on the completion of the free module generated by $\widetilde{\mathcal{P}}(H)$ with respect to $\mathcal{A}_{H}$. 
Compactness of $\mathcal{M}(x, y)$ with $\mu(x)-\mu(y)=1$ is established once bubbling off of $J$-holomorphic spheres is avoided. This follows from transversality of evaluation maps for connecting orbits and $J$-holomorphic spheres (see [15]). For a weakly monotone symplectic manifold $(M, \omega)$, Hofer and Salamon constructed Floer homology for a generic pair $(H, J)$. Here $(M, \omega)$ is called weakly monotone (or semi-positive), if the following condition holds: $\omega \cdot A<0$ for $A \in \pi_{2}(M)$ satisfying $c_{1}(M) \cdot A<3-m$, where $m$ is half of the dimension of $M$.

Denote by $\widetilde{\mathcal{M}}_{J}(A)$ the space of $J$-holomorphic spheres representing the homology class $A \in H_{2}(M ; \mathbf{Z})$. Write $\mathcal{M}_{J}(A)=\widetilde{\mathcal{M}}_{J}(A) / P S L(2, \mathbf{C})$ the moduli space of $J$-holomorphic spheres representing the homology class $A \in H_{2}(M ; \mathbf{Z})$. Suppose that $(M, \omega)$ is weakly monotone. If a sequence $f_{k}$ is not convergent in $\mathcal{M}_{J}(A)$, it converges locally uniformly outside of finitely many points. $J$-holomorphic bubbles appear around these points after a rescaling procedure. So the limit of $f_{k}$ consists of finitely many $J$-holomorphic spheres $g_{1}, \ldots, g_{l}$ satisfying the following conditions.

1. $\sum_{i=1}^{l}\left[g_{i}\right]=A$.

2. The union of images of $g_{i}$ is connected.

If the linearization operator of the $J$-holomorphic curve equation at $g_{i}$ is surjective, then $\mathcal{M}_{J}\left(\left[g_{i}\right]\right)$ is a manifold near $g_{i}$ by the implicit function theorem and its dimension is given by the Atiyah-Singer index formula.

$$
\operatorname{dim} \mathcal{M}_{J}\left(\left[g_{i}\right]\right)=2 m+2 c_{1}(T M)\left[g_{i}\right]-6 .
$$

Here 6 is the dimension of the automorphi sm group $P S L(2, \mathbf{C})$ of $\mathbf{C} P^{1}$. If evaluation maps for $\left[g_{i}\right]$ are transver sal, then the dimension of the set of limit points of $\mathcal{M}_{J}(A)$ is less than the dimension of $\mathcal{M}_{J}(A)$ at least by 2 . This insures the existence of a good compac tification. We explain this fact in the case $l=2$ for simplicity. The general case is also treated in the same spirit, but is more complicated.

Let $e v_{i}: \widetilde{\mathcal{M}}_{J}\left(\left[g_{i}\right]\right) \rightarrow M$ be the evaluation map at the point $[1,0]$ of $\mathbf{C} P^{1}$. If $e v_{1}$ and $e v_{2}$ are transversal, then the dimension of $\left(e v_{1} \times e v_{2}\right)^{-1}\left(\Delta_{M}\right)$ is $2 m+2 c_{1}(T M)\left[g_{1}\right]+$ $2 m+2 c_{1}(T M)\left[g_{2}\right]-2 m=2 m+2 c_{1}(A)$, where $\Delta_{M}$ is the diagonal set in $M \times M$. This is the space of pairs of $J$-holomorphic spheres representing $\left[g_{i}\right]$ respectively and coinciding at $[1,0]$. Then the product of two copies of the automorphism group of $\left(\mathbf{C} P^{1},[1,0]\right)$ acts on it locally freely if $\left[g_{i}\right] \neq 0$ for $i=1,2$.

This group is $2 \times 4=8$ dimensional and the moduli space of such pairs is $2 m+$ $2 c_{1}(T M)[A]-8$, which is less than $\operatorname{dim} \mathcal{M}_{J}(A)$ by 2 . McDuff proved surjectivity of the linearization operator for somewhere injective $J$-holomorphic curves with respect to a generic $J$. Here a map $f$ is called somewhere injective if there exists a point $x$ in the domain such that $d f$ is injective at $x$ and $f^{-1}(f(x))=\{x\}$. For transversality of evaluation maps, see [24]. For connecting orbits and $J$-holomorphic bubbles, a similar argument holds [15].

If a $J$-holomorphic map $f$ from a closed Riemann surface is not somewhere injective, it factors into a branched covering between closed Riemann surfaces and a somewhere injective map $h$. If $(M, \omega)$ is weakly monotone, we have $c_{1}(T M)[f] \geq c_{1}(T M)[h]$. So if a 
multi-covered curve $f$ appears as $g_{l}$, say, then the union of the image of $g_{1}, \ldots, g_{l-1}, h$ is also connected.

This condition enables us to compactify $\mathcal{M}_{J}(A)$ by attaching lower dimensional strata. We encounter a problem when a multi-covered curve $f$ satisfies $c_{1}(T M)[f]<c_{1}(T M)[h]$, i.e., $c_{1}(T M)[h]<0$. We call such a problem a "negative multiple cover problem". We shall explain the way to overcome it in the next section.

3. Kuranishi structure and multi-valued perturbation. This section is a survey on joint work with Kenji Fukaya concerning Gromov-Witten invariants and the Arnold conjecture for general symplectic manifolds [12]. There are several mathematicians who have discussed this problem independently. J. Li and G. Tian constructed Gromov-Witten invariants for algebraic manifolds and also for general symplectic manifolds. G. Liu and G. Tian used their method to prove the Arnold conjecture. There are also works by Y. Ruan, B. Siebert, and H. Hofer and D. Salamon. Gromov-Witten invariants for algebraic manifolds were also constructed by K. Behrend [3] .

To deal with compactification of the moduli space of $J$-holomorphic curves and the moduli space of connecting orbits, we introduce the notion of stable maps due to Kontsevich [16]. It is widely known that singular Riemann surfaces with at most double points are necessary to compactify the moduli space of Riemann surfaces. We need to consider the moduli space of Riemann surfaces with marked points, namely the space of $\left(C, x_{1}, \ldots, x_{k}\right)$ where $C$ is a Riemann surface and $x_{1}, \ldots, x_{k}$ are points on $C$, i.e., marked points. The genus of a singular Riemann surface $C=\bigcup \Sigma_{i}$ with at most double points is defined as the sum of the genus of its normalization and the first Betti number of a graph associated to it. Here the vertices of the graph are irreducible components. For double points, we put an edge joining vertices corresponding to irreducible components containing the double point. This gives a graph associated to $C$. A possibly singular Riemann surface $C$ of genus $g$ and with $k$ marked points and at most double points is called a stable curve, if each irreducible component $\Sigma_{i}$ of $C$ satisfies the following:

Either the genus of $\Sigma_{i}$ is at least 2, the number of marked points and double points on $\Sigma$ is at least 1 if its genus is 1 and at least 3 if its genus is 0 .

This condition is equivalent to the condition that the automorphism group of $C$ is finite. Here a homeomorphism $\phi: C \rightarrow C$ is an automorphism if its restriction to each irreducible component is biholomorphic and $\phi$ preserves marked points. The moduli space of stable curves of genus $g$ and with $k$ marked points is a compact orbifold.

When we consider the limit of a sequence of $J$-holomorphic curves with varying complex structure on the domain, we have to take the limit of complex structures on the domain into account. However this is not enough. For instance, regular fibers of an elliptic surface are holomorphic curves of genus 1. Singular fibers are limits of such curves and classified by Kodaira. In the list of singular fibers, there are "unstable curves". Kontsevich considered a stability condition for $J$-holomorphic curves.

Let $C=\sum \Sigma_{i}$ be a singular Riemann surface with at most double points. A $J$ holomorphic map $f: C \rightarrow M$ is called a stable map, if each irreducible component satisfies one of the following: 
1. The restriction of $f$ to $\Sigma_{i}$ is not a constant map.

2. The genus of $\Sigma_{i}$ is at least 2 .

3. The number of marked points and double points on $\Sigma$ is at least 1 if its genus is 1 and at least 3 if its genus is 0 .

A biholomorphic automorphism $\phi$ of $\left(C, x_{1}, \ldots, x_{k}\right)$ is called an automorphism of $f: C \rightarrow M$, if $f=f \circ \phi$ holds. The condition of stability for $f$ is, again, equivalent to the condition that its automorphism group is finite. We can introduce a topology on the moduli space of stable curves and show that it is compact and Hausdorff. A similar construction can be done for connecting orbits in Floer theory.

This space may be pathological and we may not define its fundamental class. We describe this space by "local defining equations" and "perturbing the equations" so that the zero locus becomes nice. The first step is based on the Kuranishi method [17] and the gluing argument for $J$-holomorphic curves in the appendix of [24]. The second step is subtle since usual perturbation may not work. This is because of the existence of automorphisms. We introduced the notion of multi-valued perturbation and making the zero locus nice.

Recall that a map $f:(\Sigma, j) \rightarrow(M, J)$ is $(j, J)$-holomorphic or simply $J$-holomorphic if it satisfies

$$
d f+J \circ d f \circ j=0 .
$$

Consider the left hand side of this equation as a section $\bar{\partial}$ of a Banach bundle $\mathcal{E}$ with fiber at $f$ being $\Gamma\left(\Sigma, T_{0,1}^{*} \Sigma \otimes_{\mathbf{C}} f^{*} T^{1,0} M\right)$. For fixed $j$ and $J, \bar{\partial}$ is a Fredholm section. Locally we can choose a finite dimensional subbundle $F_{f}$ in $\mathcal{E}$ such that $\bar{\partial}$ is transversal to $F_{f}$. Then the inverse image of $F_{f}$ is a finite dimensional manifold $V_{f}$. In fact, we may have non-trivial automorphisms of $f$. In this case, we can choose the subbundle so that it is invariant under the automorphism group $\Gamma_{f}$, i.e., an equivariant vector bundle over $V_{f}$. A stability condition for $f$ is finiteness of $\Gamma_{f}$. On $V_{f}$, the section $\bar{\partial}$ takes values in $F_{f}$ and gives a section $s_{f}$ of $F_{f} \rightarrow V_{f}$. For a stable map $f: C \rightarrow M$, we modify the gluing argument in [24] in the situation with "obstruction bundles" and give a local description of the moduli space in a similar way. The moduli space is locally isomorphic to the image of the zero locus of $s_{f}$ in the quotient space $U_{f}=V_{f} / \Gamma_{f} .\left(U_{f}\right.$ is an orbifold.) If $s_{f}$ is perturbed to a section transversal to the zero section in equivariant way, the zero locus in $U_{f}$ becomes an orbifold. However such a perturbation is not always possible.

So we consider a multi-valued section, each branch of which is transversal to the zero section. We can perturb $s_{f}$, not necessarily in an equivariant way, so that it is transversal to the zero section (Thom's transversality theorem). Then we take all the images of this section under $\Gamma_{f}$. Consider the set of points where one of branches of the multi-section vanishes. For a point in this set, we associate the weight by the ratio of the number of branches vanishing at the point to the number of all the branches. This construction is local and we have to make such multi-valued perturbations everywhere in a compatible way. This is discussed in terms of Kuranishi structure defined below.

A Kuranishi structure of dimension $n$ on a compact metrizable space $Y$ is a collection $\left(U_{p}, E_{p}, s_{p}, \psi_{p}, \phi_{p q}, \hat{\phi}_{p q}\right)$ for each $p \in Y$ such that 
1. $U_{p}=V_{p} / \Gamma_{p}$ is an orbifold and $E_{p}$ is an orbibundle on it.

2. $s_{p}$ is a single valued section of $E_{p}$.

3. $\psi_{p}$ is a homeomorphism from $s_{p}^{-1}(0)$ to a neighborhood of $p$ in $Y$.

4. Let $q \in \psi_{p}\left(s_{p}^{-1}(0)\right)$. Then there exists an embedding $\phi_{p q}: U_{q} \rightarrow U_{p}$ in the category of orbifolds, which is covered by an embedding of orbibundles $\hat{\phi}_{p q}: E_{q} \rightarrow E_{p}$.

5. $s_{p} \circ \phi_{p q}=\hat{\phi}_{p q} \circ s_{q}, \psi_{p} \circ \phi_{p q}=\psi_{q}$.

6. If $r \in \psi_{q}\left(s_{q}^{-1}(0)\right)$, then $\phi_{p q} \circ \phi_{q r}=\phi_{p r}, \hat{\phi}_{p q} \circ \hat{\phi}_{q r}=\hat{\phi}_{p r}$.

7. $\operatorname{dim} U_{p}-\operatorname{rank} E_{p}=n$ for all $p \in Y$.

We can find multi-valued sections for $E_{p}$ in a compatible way so that each branch is transversal to the zero section (see [12]). The last condition in the definition of Kuranishi structure is satisfied in our case, because the linearization operator of the $J$-holomorphic curve equation is a Fredholm operator and its index is invariant under compact perturbation. Note that for a higher dimensional complex manifold $Z$ and a holomorphic vector bundle $E$, the difference of dimensions of $H^{0}(Z ; E)$ and $H^{1}(Z ; E)$ is not necessarily invariant under deformation. Only the alternating sum of the dimensions of $H^{k}(Z ; E)$ is given by the Riemann-Roch-Hirzebruch formula or the Atiyah-Singer index formula and topological invariants.

We can also define an orientation of a Kuranishi structure and show that there is a canonical orientation for the Kuranishi structure on the moduli space of $J$-holomorphic curves. This enables us to define the "fundamental cycle" with rational coefficients. This is the way to overcome the negative multiple bubble problem and we can define GromovWitten invariants.

The construction of Floer homology also follows by a similar argument and we get the following:

THEOREM. Let $(M, \omega)$ be a closed symplectic manifold and $\phi$ an exact symplectomorphsm of $(M, \omega)$ with only non-degenerate fixed points. Then the number of fixed points of $\phi$ is at least the sum of Betti numbers of $M$, i.e., $\sum_{k} \operatorname{dim} H^{k}(M ; \mathbf{Q})$.

\section{References}

[1] M. Atiyah, V. Patodi and I. Singer, Spectral asymmetry and Riemannian geometry, I, Math. Proc. Cambridge Phil. Soc. 77 (1975), 43-69, II, ibid. 78 (1975), 405-432.

[2] V. I. Arnold, Mathematical Methods in Classical Mechanics, Graduate Text in Mathematics 60, Springer Verlag.

[3] K. Behrend, Gromov-Witten invariants in algebraic geometry, Invent. Math. (1997).

[4] M. Chaperon, Une idée du type géodesiques brisées, Comptes Rendues Paris 298 (1984), 293-296.

[5] Y. Chekanov, Hofer's symplectic energy and Lagrangian intersections, in: Contact and Symplectic Geometry, ed. by C. B. Thomas, Cambridge University Press, 1996; Lagrangian intersections, symplectic energy and areas of holomorphic curves, preprint.

[6] C. Conley and E. Zehnder, The Birkhoff-Lewis fixed point theorem and a conjecture of V. I. Arnold, Invent. Math. 73 (1983), 33-49; Morse type index theory for flows and periodic solutions for Hamiltonian systems, Comm. Pure Appl. Math. 37 (1984), 207-253. 
[7] A. Floer, Morse theory for lagrangian intersections, Journ. Differ. Geom. 28 (1988), 513-547; The unregularized gradient flow of the symplectic action, Comm. Pure Appl. Math. 41 (1988), 775-813; A relative Morse index for the symplectic action, Comm. Pure Appl. Math. 41 (1988), 393-407; Witten's complex and infinite dimensional Morse theory, Journ. Differential Geom. 30 (1989), 207-221; Cup length estimate on lagrangian intersections, Comm. Pure Appl. Math. 42 (1989), 335-357.

[8] A. Floer, Holomorphic spheres and symplectic fixed points, Comm. Math. Phys. 120 (1989), 575-611.

[9] A. Floer and H. Hofer, Coherent orientations for periodic orbits problems in symplectic geometry, Math. Z. 212 (1993), 13-38.

[10] A. Floer, H. Hofer and D. Salamon, Transversality in elliptic Morse theory for the symplectic action, Duke Math. Journ. 80 (1995), 251-292.

[11] K. Fukaya, The symplectic S-cobordism conjecture: a summary, in: Geometry and Physics, ed. by J. E. Andersen, J. Dupont, H. Pedersen and A. Swann, Lecture Notes in Pure and Appl. Math. 184 (1997), 209-219.

[12] K. Fukaya and K. Ono, Arnold conjecture and Gromov-Witten invariant for general symplectic manifolds (announcement); Arnold conjecture and Gromov-Witten invariant, preprint, 1996.

[13] M. Gromov, Pseudoholomorphic curves in symplectic manifolds, Invent. Math. 82 (1985), 307-347.

[14] H. Hofer, Lagrangian embeddings and critical point theory, Ann. Inst. H. Poincaré, Anal. Non Linéaire 2 (1985), 407-462; Lusternik-Schnirelmann theory for Lagrangian intersections, ibid. 5 (1988), 465-499.

[15] H. Hofer and D. Salamon, Floer homology and Novikov rings, Floer memorial volume, ed. by H. Hofer, C. Taubes, A. Weinstein and E. Zehnder, 483-524, Birkhäuser 1995.

[16] M. Kontsevich, Enumeration of rational curves by torus actions, in: Moduli space of curves, ed. by H. Dijkgraaf, C. Faber, G. v. d.Geer, 335-368, Progress in Mathematics 129, Birkhäuser 1995.

[17] M. Kuranishi, New proof for the existence of locally free complete families of complex structures, in: Conference on Complex Analysis, 1996 Mineapolis, Springer-Verlag.

[18] F. Laudenbach and J.-C. Sikorav, Persistence d'intersection avec la section nulle au cours d'une isotopie hamiltonienne dans un fibré cotangent, Invent. Math. 82 (1985), 349-358.

[19] H. V. Lê and K. Ono, Symplectic fixed points, the Calabi invariant and Novikov homology, Topology 34 (1995), 155-176.

[20] H. V. Lê and K. Ono, Cup-length estimate for symplectic fixed points, in: Contact and Symplectic Geometry, 268-295, ed. by C. B. Thomas, Publication of the Newton Institute, Cambridge Univ. Press, 1996.

[21] J. Li and G. Tian, Virtual moduli cycles and Gromov-Witten invariants for general symplectic manifolds, preprint 1996.

[22] G. Liu and G. Tian, preprint 1996.

[23] D. McDuff, Elliptic methods in symplectic geometry, Bull. Amer. Math. Soc. 23 (1990), 311-358.

[24] D. McDuff and D. Salamon, J-holomorphic curves and quantum cohomology, University Lecture Ser., 6, Amer. Math. Soc. 1994.

[25] Y. G. Oh, Floer cohomology of Lagrangian intersections and pseudo-holomorphic disks, I, Comm. Pure Appl. Math. 46 (1993), 949-994, II ibid. 46 (1993), 995-1012, III, in: 
Floer Memorial Volume, Birkhäuser 1995.

[26] K. Ono, On the Arnold conjecture for weakly monotone symplectic manifolds, Invent. Math. 119 (1995), 519-537.

[27] S. Piunikhin, D. Salamon and M. Schwarz, Symplectic Floer-Donaldson theory and quantum cohomology, in: Contact and Symplectic Geometry, ed. by C. B. Thomas, Cambridge Univ. Press 1996.

[28] Y. Ruan, Virtual neighborhoods and pseudoholomorphic curves, preprint 1996.

[29] D. Salamon, Morse theory, the Conley index and Floer homology, Bull. London Math. Soc. 22 (1990), 113-140.

[30] D. Salamon and E. Zehnder, Morse theory for periodic solutions of Hamiltonian systems and the Maslov index, Comm. Pure Appl. Math. 45 (1992), 1303-1360.

[31] M. Schwarz, Quantum cup-length estimate for symplectic fixed points, preprint 1996.

[32] M. Schwarz, Morse homology, Progress in Math. 111, Birkhäuser, 1993.

[33] B. Siebert, Gromov-Witten invariants for general symplectic manifolds, preprint 1996. 